\title{
Adiponectin, Adiponectin Receptors, and Epigenetic Regulation of Adipogenesis
}

\author{
T. Kadowaki, T. Yamauchi, H. Waki, M. Iwabu, M. Okada-Iwabu, and M. NaKamura \\ Department of Diabetes and Metabolic Diseases, Graduate School of Medicine, \\ University of Tokyo, Tokyo, Japan \\ Correspondence: kadowaki-3im@h.u-tokyo.ac.jp
}

\begin{abstract}
We studied the molecular mechanism of obesity-induced insulin resistance and adipogenesis. Plasma adiponectin and adiponectin receptor (AdipoR1) in muscle are down-regulated in obesity. Analysis of muscle-specific AdipoR1 knockout mice revealed the pivotal role of adiponectin/AdipoR1 in the regulation of mitochondrial biogenesis via AMPK- and SIRT1mediated PGC- $1 \alpha$ activation as well as $\mathrm{Ca}^{2+}$-dependent up-regulation of PGC- $1 \alpha$ expression. Reduced adiponectin/AdipoR1 signals in muscle in obesity appear to cause PGC-1 $\alpha$ inactivation as well as down-regulation and consequently impaired mitochondrial biogenesis and insulin resistance. In the epigenetic analysis of adipogenesis, we demonstrated that adipocytespecific formaldehyde-assisted isolation of regulatory elements (FAIRE) peaks are associated with genes up-regulated by adipogenesis, whereas preadipocyte-specific FAIRE peaks are associated with genes down-regulated by adipogenesis. Computational motif analyses of adipocyte-specific FAIRE peaks confirmed PPAR $\gamma$ and CCAAT-enhancer binding proteins (C/EBPs) on the top list, consistent with their crucial roles in adipogenic transcription, and also revealed NFIA and NFIB to be important regulators of proper adipocyte differentiation.
\end{abstract}

Obesity is increasing worldwide and causes various diseases such as diabetes, cardiovascular disease, and some forms of cancer. To determine the molecular causes of these obesity-linked diseases, we have been working on adiponectin, an adipokine that is most abundantly expressed in white adipose tissue and becomes downregulated in obesity to develop various obesity-related diseases. We have also been working on adipogenesis itself with the hope that the identification of the molecular mechanism of adipogenesis may shed light on the causes of obesity and obesity-related diseases.

\section{ADIPONECTIN AND ADIPONECTIN RECEPTORS}

Adiponectin (Scherer et al. 1995; Hu et al. 1996; Maeda et al. 1996; Nakano et al. 1996) is an adipokine that protects against diabetes and atherosclerosis. Plasma adiponectin levels are decreased in obesity, insulin resistance, and type 2 diabetes (Kadowaki et al. 2006). Administration of adiponectin has been shown to elicit glucoselowering effects and ameliorate insulin resistance (Berg et al. 2001; Yamauchi et al. 2001). Adiponectin knockout mice exhibit insulin resistance and diabetes (Kubota et al. 2002). Adiponectin has also been shown to increase fatty acid oxidation by stimulation of AMP-activated protein kinase (AMPK) (Yamauchi et al. 2002) and also by peroxisome proliferator-activated receptor $\alpha(\operatorname{PPAR} \alpha)$ (Yamauchi et al. 2003a).

We previously reported the cloning of complementary DNAs encoding adiponectin receptors (AdipoR) 1 and 2 by expression cloning (Yamauchi et al. 2003b). Liver expresses both AdipoR1 and AdipoR2, whereas skeletal muscle expresses predominantly AdipoR1. Both receptors are predicted to contain seven transmembrane domains (Yamauchi et al. 2003b) but to be structurally and functionally distinct from G-protein-coupled receptors (GPCRs). Adiponectin receptors may thus comprise a new receptor family (Fig. 1).

Using AdipoR1 and/or AdipoR2 knockout mice, we previously showed that AdipoR1 and AdipoR2 act as the major receptors for adiponectin in vivo and have important roles in the regulation of glucose metabolism and insulin sensitivity in vivo (Yamauchi et al. 2007). Importantly, obesity decreases not only plasma adiponectin but also adiponectin receptors in liver and skeletal musle to cause adiponectin resistance (Kadowaki et al. 2006). Moreover, in the liver, AdipoR1 activates the AMPK pathway to suppress gluconeogenesis and lipogenesis, and AdipoR2 activates the PPAR $\alpha$ pathway to stimulate fatty acid oxidation and suppress inflammation and oxidative stress (Yamauchi et al. 2007). In skeletal muscle, insulin resistance has been reported to be associated with mitochondrial dysfunction (Patti et al. 2003; Petersen et al. 2004). However, the exact cause of mitochondrial dysfunction has yet to be clarified. To clarify whether decreased adiponectin/AdipoR1 signaling could be associated with mitochondrial dysfunction, we analyzed muscle-specific AdipoR1-knockout (muscle-R1KO) mice (Iwabu et al. 2010) and attempted to determine the molecular mechanism whereby adiponectin/AdipoR1 would exert their biological effects. 


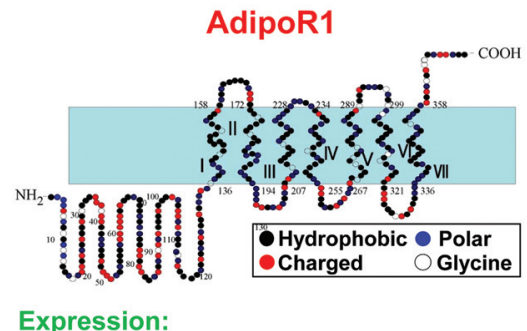

Expression:

Muscle, liver, hypothalamus, $M \varphi$ etc.

Function:

\section{AMPK activation}

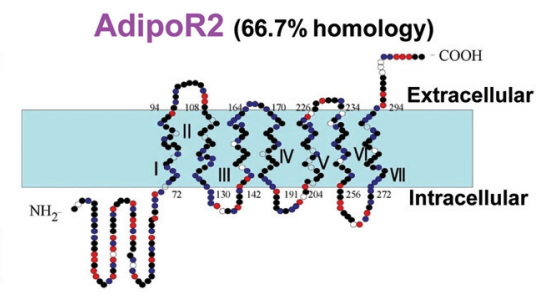

Liver, vasculature, etc.

PPAR activation

Figure 1. Cloning of and functional analysis of adiponectin receptors. Adiponectin receptors AdipoR1 and AdipoR2 are predicted to be integral membrane proteins with seven transmembrane domains, whose amino terminus is internal and carboxyl terminus is external opposite to G-protein-coupled receptors (GPCRs) (Yamauchi et al. 2003b, 2007).

\section{Decreased PGC-1 $\alpha$ and Mitochondria in Muscle-R1KO}

Muscle-R1KO mice showed decreased phosphorylation of AMPK. Moreover, the administration of adiponectin increased the phosphorylation of AMPK in the muscle of control littermates but not in that of muscle-R1KO mice, whereas adiponectin increased the phosphorylation of AMPK in the liver of both genotypes. Muscle-R1KO mice also exhibited decreased molecules involved in mitochondrial biogenesis, such as PGC- $1 \alpha$, at both messenger RNA and protein levels. Adiponectin increased the expression levels of PGC- $1 \alpha$ in muscle of control littermates but not in that of muscle-R1KO mice. Muscle$\mathrm{R} 1 \mathrm{KO}$ mice showed decreases in molecules involved in mitochondrial biogenesis such as oestrogen-related receptor $\alpha$ (Esrr $\alpha)$, molecules involved in transcription such as nuclear respiratory factor 1 (Nrf1), and molecules involved in mitochondrial DNA replication/translation such as mitochondrial transcription factor A (Tfam) (Iwabu et al. 2010). Moreover, the expression levels of several oxidative phosphorylation and other mitochondrial genes as well as mitochondrial content were significantly reduced in muscle-R1KO mice. Mitochondrial function was also decreased in muscle-R1KO mice (Iwabu et al. 2010). In soleus muscle of muscle-R1KO mice, type I fibers were reduced by $20 \%$. To study the effect of AdipoR1 ablation on skeletal muscle function, we challenged control and muscle-R1KO mice with involuntary physical exercise and assessed muscle endurance during treadmill running. Muscle endurance was significantly lower for muscle-R1KO mice than control mice (Iwabu et al. 2010). We next examined the expression of metabolic genes and found that molecules involved in fatty acid oxidation were significantly decreased in muscle-R1KO mice, which was associated with decreased $\beta$ oxidation and increased triglyceride content in skeletal muscle. Muscle-R1KO mice exhibited decreased expression levels of mitochondrial and cytoplasmic oxidative-stress-detoxifying genes such as manganese superoxide dismutase (Sod2) and catalase (Cat), respectively, and increased oxidative stress such as thiobarbituric acid reactive substance (TBARS) in muscle (Iwabu et al. 2010).

\section{Insulin Resistance in Muscle-R1KO Mice}

Upon glucose administration, plasma glucose and insulin levels were significantly higher in muscle-R1KO mice than in control mice, suggesting that muscleR1KO mice have insulin resistance (Fig. 2). We performed a hyperinsulinemic-euglycemic clamp experiment. Disruption of AdipoR1 in muscle did not significantly alter endogenous glucose production, whereas it significantly decreased the glucose disposal rate and the glucose infusion rate, indicating decreased insulin sensitivity in muscle (Iwabu et al. 2010).

\section{Adiponectin Induces $\mathrm{Ca}^{2+}$ Influx by AdipoR1}

To determine molecular mechanisms of muscle phenotypes of AdipoR1 knockout mice, we used $\mathrm{C}_{2} \mathrm{C}_{12}$ myocytes. Interestingly, treatment of $\mathrm{C}_{2} \mathrm{C}_{12}$ myocytes with adiponectin resulted in an increase in the intracellular $\mathrm{Ca}^{2+}$ concentration measured by fura-2-based fluorescent imaging (Iwabu et al. 2010). Removal of extracellular free $\mathrm{Ca}^{2+}$ by EGTA almost completely abolished the adiponectin-induced $\mathrm{Ca}^{2+}$ response. Suppression of AdipoR1 expression using a specific siRNA largely reduced the calcium response of $\mathrm{C}_{2} \mathrm{C}_{12}$ myocytes to adiponectin. These results indicate that the influx of extracellular $\mathrm{Ca}^{2+}$ after adiponectin treatment of $\mathrm{C}_{2} \mathrm{C}_{12}$ myocytes is mediated by AdipoR1 (Iwabu et al. 2010). Incubation of $\mathrm{C}_{2} \mathrm{C}_{12}$ myocytes with adiponectin increased the phosphorylation of the AMPK $\alpha$ subunit at Thr 172, and EGTA partially suppressed adiponectin-induced increased AMPK phosphorylation, suggesting that adiponectin stimulates AMPK phosphorylation in part via a $\mathrm{Ca}^{2+}$-dependent fashion. We also examined whether adiponectin-induced $\mathrm{Ca}^{2+}$ influxes into skeletal muscle might be impaired in muscle-R1KO mice through in vivo imaging. We found that, whereas treatment of soleus muscle with adiponectin resulted in an increase in the intracellular $\mathrm{Ca}^{2+}$ 

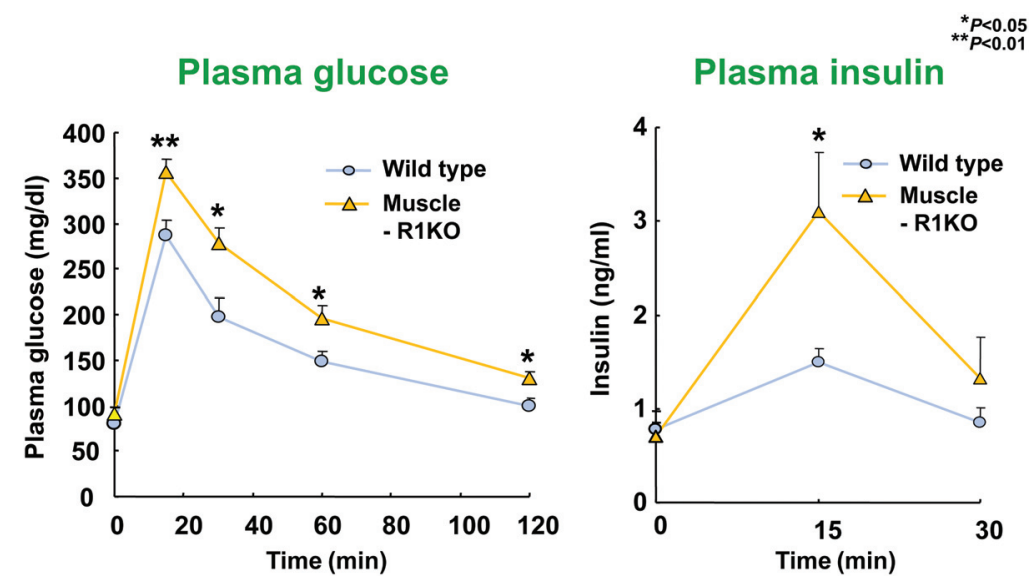

Figure 2. Muscle-specific AdipoR1 knockout mice showed insulin resistance and hyperglycemia. (Modified from Iwabu et al. 2010; (C) Macmillan.)

concentration as measured by fura-2-based fluorescent imaging in control mice, muscle-R1KO mice almost completely abolished the calcium response of soleus muscle to adiponectin, consistent with the observation that adiponectin significantly increased the phosphorylation of CaMKI, an intracellular substrate of CaMKK $\beta$, by AdipoR1 in skeletal muscle in vivo. We also attempted to examine whether simultaneous activation of $\mathrm{Ca}^{2+}$ signaling and AMPK/SIRT1 pathways by exercise, independent of AdipoR1, could rescue phenotype in muscle-R1KO mice. Two weeks of exercise significantly ameliorated insulin resistance and increased mitochondrial content and function such as citrate synthase activities in muscles of muscle-R1KO mice (Iwabu et al. 2010).

Here, we provide evidence that muscle-R1KO mice exhibit decreased mitochondrial content and enzymes. PGC- $1 \alpha$ is a key regulator of mitochondrial content and function (Wu et al. 1999; Mootha et al. 2003, 2004; Handschin and Spiegelman 2008). We have demonstrated that muscle-R1KO mice exhibit decreased PGC- $1 \alpha$ expression as well as decreased deacetylation and activation of PGC-1 $\alpha$ (data not shown) (Iwabu et al. 2010). Thus, adiponectin induced $\mathrm{Ca}^{2+}$ influx via AdipoR1, thereby activating CaMKK $\beta$, which led to increased PGC- $1 \alpha$ expression. Moreover, adiponectin/ AdipoR1 activated AMPK and SIRT1, thereby inducing PGC-1 $\alpha$ deacetylation and activation (Fig. 3). These data indicate that adiponectin and AdipoR1 stimulate increases in both the expression and activation of PGC- $1 \alpha$, in a similar fashion to exercise. Although the degree of decreased PGC- $1 \alpha$ expression was approximately $40 \%$ in muscle-R1KO mice, the extent of decreased mitochondrial biogenesis and decreased exercise endurance was comparable to that observed for muscle-specific PGC$1 \alpha$-knockout mice, which may be explained by the finding that adiponectin and AdipoR1 increase not only PGC- $1 \alpha$ expression but also PGC- $1 \alpha$ activity. Importantly, decreases in the expression of PGC-1 $\alpha$ and downstream mitochondrial genes were also observed in type 2 diabetic patients and individuals at increased risk of developing diabetes owing to their family history (Mootha et al. 2003, 2004; Patti et al. 2003) as well as in obese

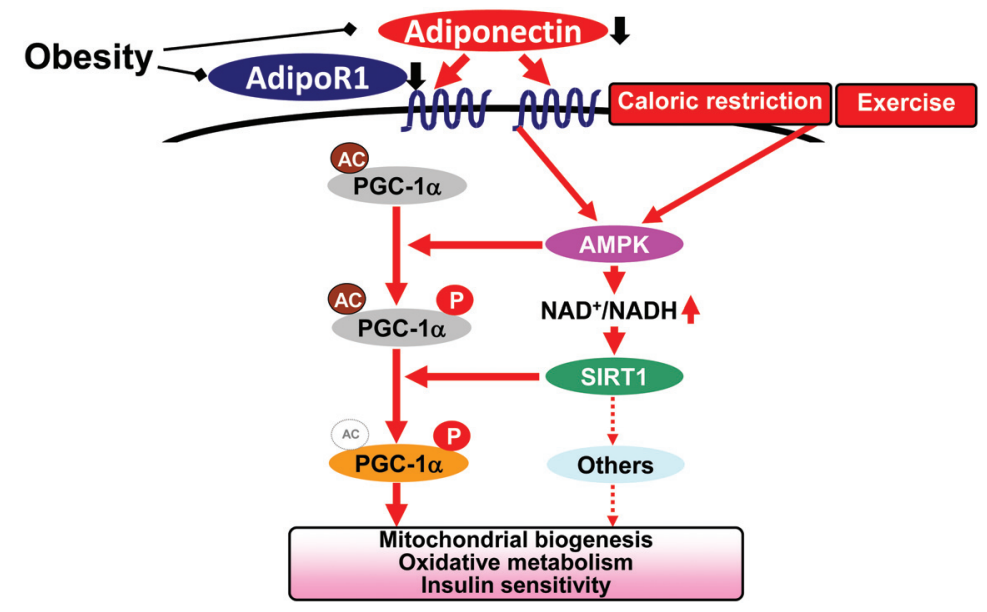

Figure 3. Adiponectin via AdipoR1 activates AMPK-SIRT1-PGC-1 $\alpha$ in muscle. (Modified from Iwabu et al. 2010; CMacmillan; see also Cantó et al. 2009, 2010.) 
diabetic $d b / d b$ mice (Iwabu et al. 2010). These data indicate that decreased adiponectin/AdipoR1 signals in muscle in pathophysiological conditions such as obesity may have causal roles in the development of PGC- $1 \alpha$ dysregulation and mitochondrial dysfunction.

In skeletal muscle, AdipoR1 regulates insulin sensitivity by several mechanisms. First is the activation of S6K1, which has been reported to be able to cause insulin resistance by increased phosphorylation of Ser 636/639 in IRS-1. S6K1 is significantly inhibited by AMPK. In skeletal muscle of muscle-R1KO mice, AMPK activation was reduced and activation of S6K1 and phosphorylation of Ser 636/639 in IRS-1 was indeed increased (Iwabu et al. 2010). Second is increased oxidative stress, which has been causally linked to insulin resistance by increased phosphorylation of Ser 302 in IRS-1 through JNK activation. Several oxidative stress detoxification genes are regulated by AMPK and PGC- $1 \alpha$, and the expression levels of these genes, such as Sod2 and Cat were reduced, which was associated with increased thiobarbituric acid reactive substances (TBARS) in skeletal muscle of muscle-R1KO mice (Iwabu et al. 2010). Third is increased triglyceride content, which has been associated with insulin resistance by increased phosphorylation of Ser 302 in IRS-1 through JNK activation. Molecules involved in fatty acid oxidation are regulated by AMPK and PGC- $1 \alpha$, and the expression levels of these genes, such as Mcad, were reduced, which was associated with increased triglyceride content in skeletal muscle of muscle-R1KO mice (Iwabu et al. 2010). Consistent with increased TBARS and triglyceride content, JNK activation and phosphorylation of Ser 302 in IRS-1 were indeed increased (Iwabu et al. 2010).

Exercise has been reported to have beneficial effects on longevity and lifestyle-related diseases and at the same time to activate $\mathrm{Ca}^{2+}$, AMPK, SIRT1, and PGC- $1 \alpha$ pathways. In this study, we clearly demonstrated that adiponectin and AdipoR1 regulate PGC- $1 \alpha$ and mitochondria via $\mathrm{Ca}^{2+}$ and AMPK/SIRT1 (Fig. 3). Therefore, agonism of AdipoR1 as well as strategies to increase AdipoR1 in muscle could be exercise mimetics.

In conclusion, adiponectin-AdipoR1 has crucial physiological and pathophysiological roles in muscle and is involved in the regulation of $\mathrm{Ca}^{2+}$ signaling, PGC- $1 \alpha$ expression and activation, mitochondrial function and oxidative stress, glucose and lipid metabolism, exercise endurance, and insulin resistance (Iwabu et al. 2010). This study suggests that agonism of AdipoR1, as well as strategies to increase AdipoR1 in muscle, may be logical approaches to providing a new treatment modality for mitochondrial dysfunction, insulin resistance, and type 2 diabetes linked to obesity.

\section{EPIGENETIC REGULATION OF ADIPOGENESIS}

Coordinated actions of transcription factors and cofactors on regulatory DNA sequences produce transcriptional activation of the eukaryotic gene. Therefore, identification and mapping of the genome's regulatory elements are critical for understanding how cell-typeselective regulation of genes in the genome is achieved.

Traditionally, regulatory elements have been identified by DNase I hypersensitivity assay combined with Southern blot analysis (Heintzman et al. 2009; Siersbæk et al. 2011). That assay coupled with microarray or highthroughput sequencing (DNase-ChIP or DNase-seq) was effectively applied in genome-wide identification of open chromatin regions.

To understand the molecular mechanisms of adipogenesis, which is regulated by a cascade of various transcription factors such as PPAR $\gamma$ and $\mathrm{C} / \mathrm{EBP} \alpha$, it is important to identify some upstream epigenetic regulatory mechanisms. Lieb and colleagues recently developed formaldehyde-assisted isolation of regulatory elements (FAIRE) as a simple procedure to isolate nucleosome-depleted DNA from chromatin (Fig. 4) (Giresi et al. 2007). FAIRE detects open chromatin structure in much the same way as the DNase I hypersensitivity assay, but with advantages, such as obviating the need for clean nuclei preparation and laborious enzyme titrations. Coupled with highthroughput sequencing (FAIRE-seq), FAIRE allows unbiased identification of potential regulatory elements without prior knowledge of binding factors (Waki et al. 2011).

\section{Genome-Wide Profiling of Open Chromatin Regions in 3T3-L1 Adipocytes by FAIRE-seq}

Our study took an unbiased approach to mapping adipocyte-specific regulatory elements in the genome by using FAIRE in 3T3-L1 adipocytes (on days 0 and 8 of differentiation) and NIH-3T3 fibroblasts (Fig. 4) (Waki et al. 2011). This approach identified in the genome 37,781 FAIRE peaks in 3T3-L1 on day 0 and 26,611 on day 8 , plus 36,111 in NIH-3T3 cells - all with a false discovery rate of $10^{-4}$. Using ChIP-seq analyses, we also generated genome-wide maps of binding sites for $\operatorname{PPAR} \gamma$, the master regulator of adipocyte differentiation, for $\operatorname{RXR} \alpha$, its heterodimer partner, for histone $\mathrm{H} 3$ lysine 4 trimethylation (H3K4me3), and for CCCTC-binding factor (CTCF). Figure 5 shows a representative map of results generated near Pparg, a transcription factor upregulated by differentiation and important in adipocyte differentiation (Waki et al. 2011). Consistent with previous observations, $28 \%$ of the FAIRE peaks were detected near the transcription start sites (TSSs; 6500 base pairs) and are referred to as promoter FAIRE peaks, whereas $72 \%$ were located outside known TSSs and are referred to as nonpromoter FAIRE peaks. Average profiling revealed that a FAIRE signal, H3K4me3, and histone H3 lysine 27 acetylation (H3K27ac) were observed at TSSs of actively transcribed genes (Waki et al. 2011). On the other hand, nonpromoter FAIRE peaks were accompanied by monomodal enrichment of $\mathrm{H} 3 \mathrm{~K} 4 \mathrm{mel}$ and were devoid of H3K4me3 enrichment, a condition described as the signature of enhancers (Waki et al. 2011).

We next compared the FAIRE peaks in 3T3-L1 cells on days 0 and 8 and in NIH-3T3 cells. The promoter FAIRE peaks were relatively constant among the three groups. 

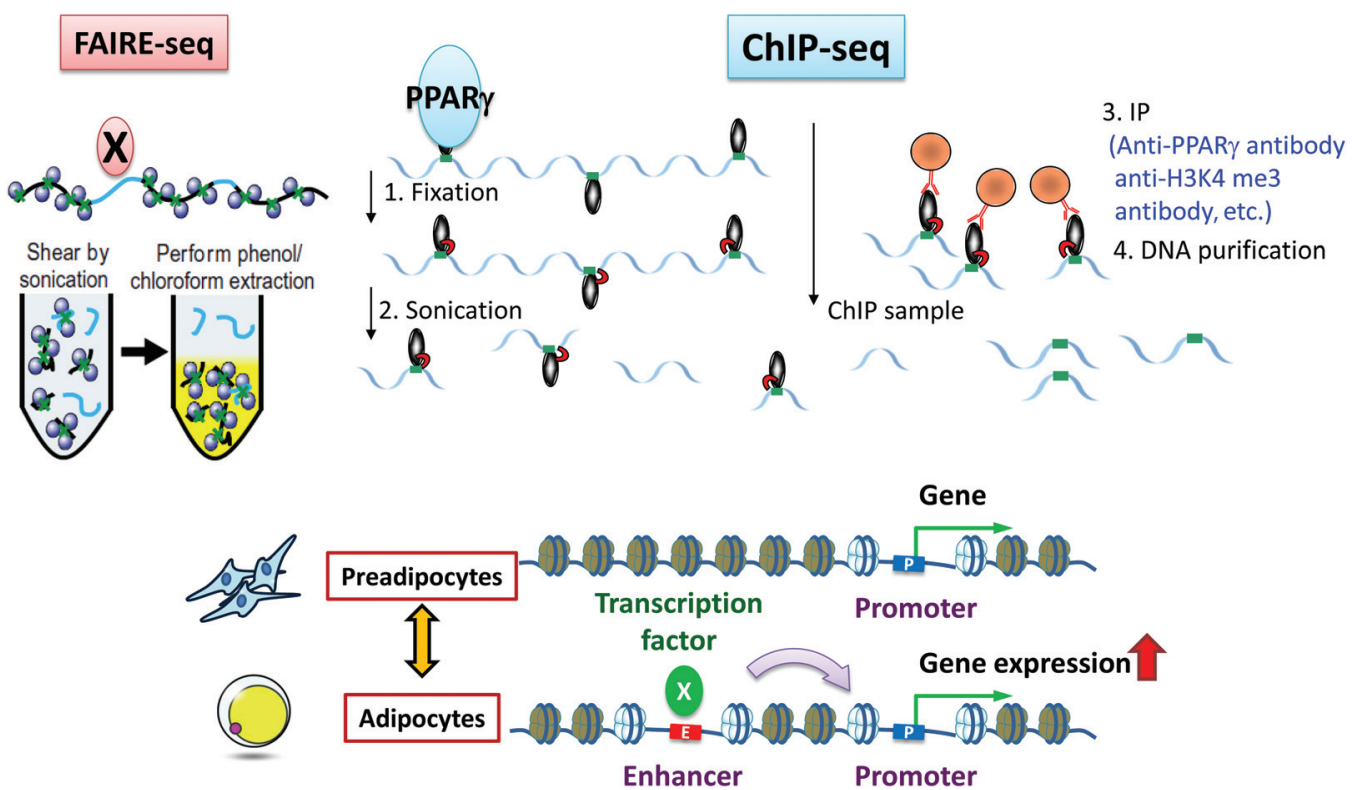

Figure 4. High-throughput sequencing formaldehyde-assisted isolation of regulatory elements (FAIRE-seq) and ChIP-seq in adipocyte differentiation (modified from Giresi et al. 2007).

More than $70 \%$ of those peaks in day 0 and day 83 T3-L1 cells and in NIH-3T3 cells were shared by all three groups. In contrast, nonpromoter FAIRE peaks showed dynamic change. The three groups shared only $25 \%$, $45 \%$, and $26 \%$ of nonpromoter FAIRE peaks, respectively, in day 0 and day 8 3T3-L1 cells and NIH-3T3 cells. Moreover, a significant proportion of the nonpromoter FAIRE peaks were cell-type specific, implying the role of nonpromoter regulatory elements in cell-type-specific transcriptional regulation (Waki et al. 2011).

To determine whether nonpromoter FAIRE peaks were functionally associated with cell-type-specific gene expression, we analyzed the relationship between the presence of the adipocyte- or preadipocyte-specific nonpromoter FAIRE peaks and the change in gene expression during adipocyte differentiation (Waki et al. 2011). Those
FAIRE peaks were enriched in the vicinity of genes, expression levels of which were highly induced or suppressed during adipocyte differentiation. Importantly, as the number of the adipocyte-specific FAIRE peaks associated with a gene increased, the fraction of up- or downregulated genes increased or decreased, respectively, whereas as the number of associated preadipocyte-specific FAIRE peaks increased, the fraction of up- or downregulated genes decreased or increased, respectively (Waki et al. 2011).

\section{Analysis of Binding Sites for PPAR $\gamma$ and RXR $\alpha$ in 3T3-L1 Adipocytes}

PPAR $\gamma$ is key regulator of adipocyte development (Nielsen et al. 2008; Tontonoz and Spiegelman 2008).

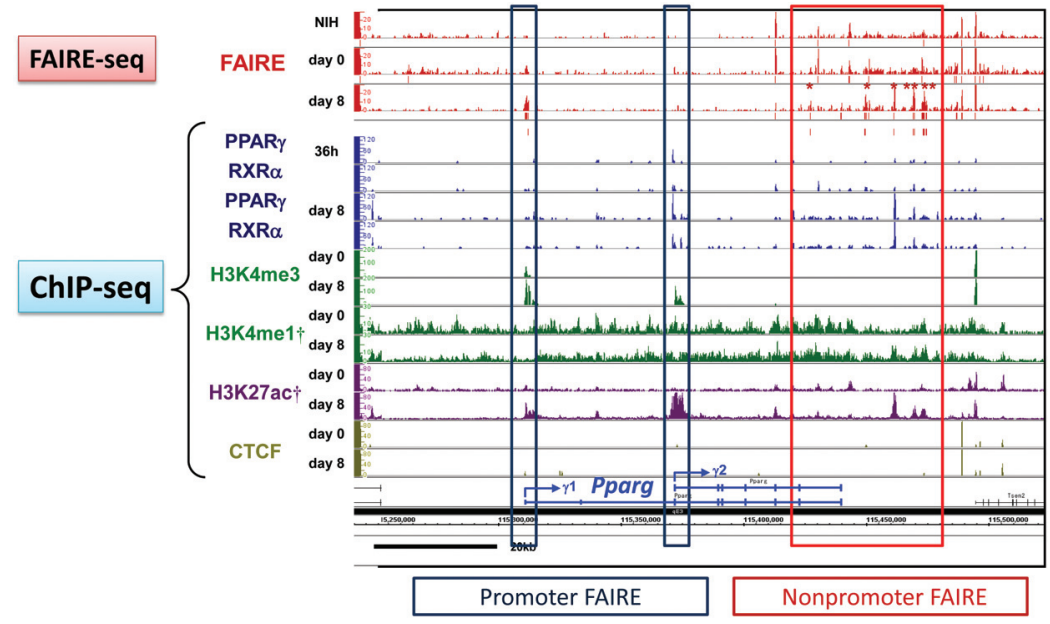

Figure 5. Pparg gene locus. (Modified from Waki et al. 2011.) 
To determine the contribution of PPAR $\gamma$ to adipocytespecific transcriptional regulation, we conducted ChIPseq analyses using antibodies specific for either PPAR $\gamma$ or RXR $\alpha$ in 3T3-L1 adipocytes at $36 \mathrm{~h}$ and 8 days after induction of differentiation (Fig. 6). The number of PPAR $\gamma$ binding sites increased during differentiation, whereas the number of $\mathrm{RXR} \alpha$ binding sites remained virtually constant. Significant overlap between the PPAR $\gamma$ and $\mathrm{RXR} \alpha$ binding sites was consistent with the heterodimer formation of PPAR $\gamma$ and $\operatorname{RXR} \alpha$ (Kliewer et al. 1992; Wakabayashi et al. 2009). To determine the contribution of PPAR $\gamma$ to the adipocyte-specific open chromatin regions, we calculated percentage fractions of the FAIRE peaks that were stratified by FAIRE signal in 3T3$\mathrm{L} 1$ on days 0 and 8 , and that overlapped either the PPAR $\gamma$ binding sites or $\mathrm{C} / \mathrm{EBP} \alpha$ binding sites in 3T3-L1 reported by Schmidt et al. (2011). Both PPAR $\gamma$ and C/EBP $\alpha$ binding sites were enriched in the fractions of adipocyte-specific FAIRE peaks, and they respectively accounted for $45.3 \%$ and $11.7 \%$ of the adipocyte-specific FAIRE peaks (Fig. 6). These data support the role of PPAR $\gamma$ and $\mathrm{C} /$ $\mathrm{EBP} \alpha$ as primary transcription factors that drive adipocyte-specific gene expression.

\section{Clustering of Multiple Adipocyte-Specific Nonpromoter FAIRE Peaks and PPAR $\gamma$ Binding Sites}

Genes that were highly induced by adipocyte differentiation often harbored multiple adipocyte-specific FAIRE peaks and/or PPAR $\gamma$ binding sites in their vicinity, as suggested by the linear correlation between the number of the associated adipocyte-specific FAIRE peaks and the robustness of the induction of the gene by adipocyte differentiation. To determine whether these multiple regions have functional regulatory elements, we selected AdipoR2 (Yamauchi et al. 2003b). Although AdipoR2 was regulated by PPAR $\gamma$ and $\operatorname{PPAR} \alpha$, conventional 22-kb promoter studies failed to identify the response element. Our ChIP-seq analysis revealed a cluster of multiple PPAR $\gamma /$ RXRa binding sites in the intron 1, downstream from the TSS of AdipoR2 (Waki et al. 2011). We iden- tified putative DR-1 motifs in these binding sites and tested them by gel-shift assay and luciferase assay. These motifs were indeed bound by the PPAR $\gamma / \mathrm{RXR} \alpha$ heterodimer and were functional in the luciferase assay, suggesting the functionality of these elements and the advantage of a genome-wide approach over the conventional "promoter-bashing" approach to identifying such response elements (Waki et al. 2011).

\section{Sequence Motif Analyses of DNA Sequences of the Adipocyte-Specific Nonpromoter FAIRE Peaks: Identification of NFI Family Transcription Factors Involved in Adipogenesis}

Next, we performed enrichment analyses of known motifs using AME in the MEME suite and the TRANSFAC (Wingender et al. 2000) and JASPER (Bryne et al. 2008) motif databases to identify motifs enriched in either adipocyte- or preadipocyte-specific FAIRE peaks compared with the background (Fig. 7). Figure 8 shows the top of the list of TRANSFAC motifs enriched in the adipocyte- and preadipocyte-specific FAIRE peaks (Waki et al. 2011). The motifs for PPAR $\gamma$ (and other DR1 motifs) and C/EBPs are on the list, consistent with their critical roles in adipogenic transcription. Motif analyses using the JASPER motif database showed enrichment of the motifs for PPAR $\gamma, C / E B P s$, and Zfp423 (Gupta et al. 2010), a recently identified adipogenic regulator.

There were several other motifs for transcription factors, their functions not previously linked to adipocyte differentiation. We focused on a motif for the NFI family of transcription factors (Waki et al. 2011). The murine NFI family consists of NFIA, NFIB, NFIC, and NFIX and was identified as a site-specific DNA binding protein that bound to the adenovirus origin of replication. It forms a dimer to bind to the symmetric consensus sequence TTGGC(N5)GCCAA. We first examined the expression change of these factors in in vitro adipocyte differentiation and found that the expressions of NFIA and NFIB were significantly induced during differentiation of 3T3L1 and another adipogenic cell line, 3T3-F442A. Consistent with this pattern, both NFIA and NFIB were highly
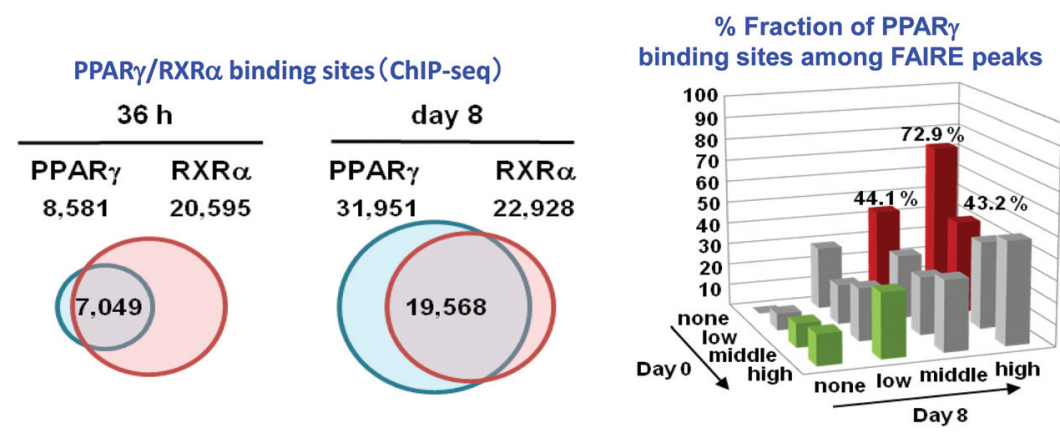

Figure 6. Comparisons between PPAR $\gamma$ binding sites (ChIP-seq) and adipocyte-enriched FAIRE peaks. RXR $\alpha$ binding was constant, whereas PPAR $\gamma$ binding increased during differentiation. Consistent with heterodimer formation of PPAR $\gamma$ and RXR $\alpha, 51 \%$ of adipocyte-enriched FAIRE peaks overlapped binding sites for PPAR $\gamma$, supporting the primary roles of PPAR $\gamma$ as a major adipogenic factor. (Modified from Waki et al. 2011.) 


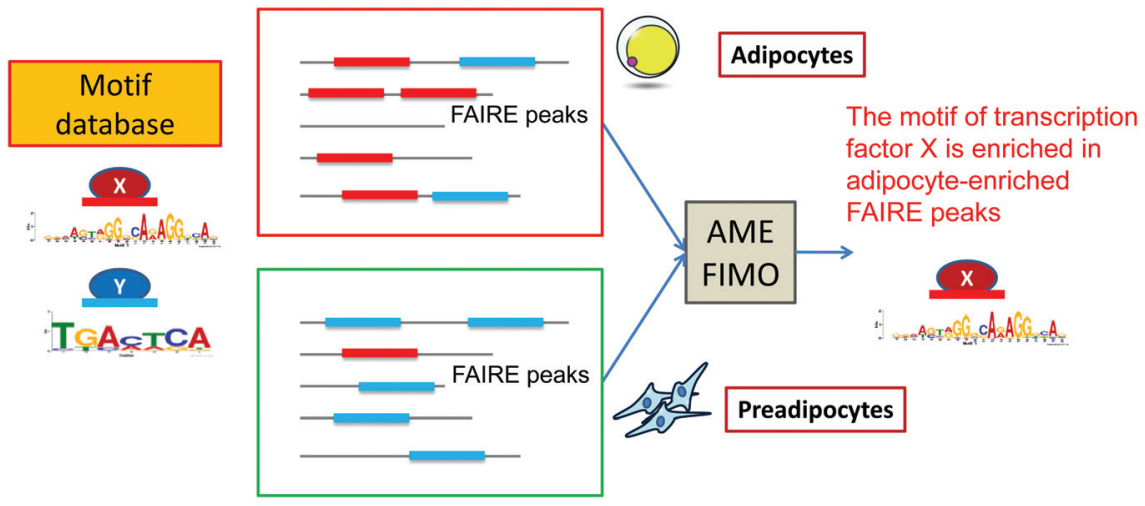

Figure 7. Motif analysis: Identification of novel factors. Known motif mapping compares frequency of binding motifs of known transcription factors between adipocyte- or preadipocyte-enriched FAIRE peaks.

expressed in a variety of adipose tissue depots in addition to the brain (Waki et al. 2011). We next examined the effect of siRNA knockdown of NFIA and NFIB on adipogenic gene regulation and adipocyte differentiation. Interestingly, induction of the expression of the adipogenic transcription factors PPAR $\gamma$ and $\mathrm{C} / \mathrm{EBP} \alpha$ and of downstream genes was significantly suppressed by siRNA knockdown of either NFIA or NFIB. Consistent with the gene expression change, we observed significant reduction of lipid accumulation as judged by oil red $\mathrm{O}$ staining, suggesting physiological roles for NFIA and NFIB in adipocyte differentiation. We confirmed the effect of NFIA and NFIB knockdown on adipogenesis using independent pooled siRNA.

We demonstrated that genome-wide mapping of open chromatin regions by FAIRE-seq is a simple, accurate method that allows a snapshot view of regulatory elements in the genome. Although open chromatin regions detected by FAIRE-seq include promoters of transcribed genes, enhancers, and insulators, open chromatin regions that vary in two different conditions probably contain regulatory elements that play roles in the specific biological process (Waki et al. 2011). By comparing open chromatin regions in preadipocytes and adipocytes, we identified the adipocyte- and preadipocyte-specific FAIRE peaks in the genome (Waki et al. 2011). Functionally, we demonstrated that the adipocyte-specific FAIRE peaks were associated with genes up-regulated by adipogenesis, whereas the preadipocyte-specific FAIRE peaks were associated with genes down-regulated by adipogenesis. Adipocyte gene expression appears to be mediated through multiple regulatory elements distal

JASPAR CORE motifs

Adipocyte-specific FAIRE peaks

\begin{tabular}{ccccc}
\hline Motif & Name & $\begin{array}{c}\text { Corrected } \\
\text { P-value }\end{array}$ & $\begin{array}{c}\text { Enrich- } \\
\text { Rent } \\
\text { Rad/pAd) }\end{array}$ \\
\hline MA0119.1 & $\begin{array}{c}\text { TLX1:: } \\
\text { NFIC }\end{array}$ & $6.9 . E-23$ & 1.55 \\
\hline MA0065.2 & $\begin{array}{c}\text { PPARG:: } \\
\text { RXRA }\end{array}$ & $3.7 . E-12$ & 2.04 \\
\hline MA0058.1 & MAX & $4.2 . E-07$ & 1.48 \\
\hline MA0114.1 & $\begin{array}{c}\text { HNF4A } \\
\text { (PPAR) }\end{array}$ & $7.3 . E-07$ & 2.09 \\
\hline MA0102.2 & CEBPA & $1.4 . E-05$ & 2.52 \\
\hline MA0116.1 & Zfp423 & $1.4 . E-05$ & 1.22 \\
\hline MA0043.1 & HLF & $1.9 . E-04$ & 1.6 \\
\hline & SP1 & $1.0 . E-02$ & 1.37 \\
\hline
\end{tabular}

Preadipocyte-specific FAIRE peaks

\begin{tabular}{ccccc}
\hline Motif & Name & $\begin{array}{c}\text { Corrected } \\
\text { P-value }\end{array}$ & $\begin{array}{c}\text { Enrich- } \\
\text { Rentio } \\
\text { (Ad/pAd) }\end{array}$ \\
\hline MA0099.2 & AP1 & $5.5 E-215$ & 0.06 \\
\hline MA0150.1 & NFE2L2 & $5.2 E-29$ & 0.37 \\
\hline MA0002.2 & RUNX1 & $5.9 E-22$ & 0.62 \\
\hline MA0090.1 & TEAD1 & $3.6 \mathrm{E}-14$ & 0.44 \\
\hline MA0117.1 & Mafb & $6.9 E-08$ & 0.66 \\
\hline MA0156.1 & FEV & $2.0 \mathrm{E}-05$ & 0.60 \\
\hline MA0047.2 & Foxa2 & $2.7 \mathrm{E}-03$ & 0.62 \\
\hline
\end{tabular}

Figure 8. Known motif analysis (AME, FIMO)_JASPAR CORE database. (Modified from Waki et al. 2011.) 
to TSSs: Greater induction of gene expression by differentiation means greater likelihood that more adipocytespecific FAIRE peaks are associated with the gene. This implies that optimal gene transcriptional regulation may require coordinated actions of multiple regulatory elements. Therefore, although valuable and informative, the proximal promoter assay may not always be sufficient (Waki et al. 2011).

Computational motif analysis is used to discover new transcription-factor binding motifs in sequences inferred from genome-wide studies such as ChIP-seq. In genomewide ChIP analysis of transcription factors, motif analysis is used to obtain their accurate binding motifs and discover unknown DNA binding factors that colocalize with the transcription factors of interest. The analyses, however, rely on prior knowledge about transcription factors and the regions to be analyzed are limited to their binding sites. In contrast, the combination of motif analyses and mapping of regulatory elements by FAIRE-seq does not require such prior knowledge and hence offers a distinct advantage in unbiased screening for novel transcription factors that are important in given biological processes. In our study, we retrieved the motifs for PPAR $\gamma$ and $\mathrm{C} /$ EBPs and for known regulators that top the list of the motifs identified in the adipocyte- or preadipocyte-specific FAIRE peaks (Waki et al. 2011). Furthermore, we demonstrated that NFIA and NFIB were functionally required for proper adipocyte differentiation (Waki et al. 2011). These results demonstrated that motif analyses of cell-type-specific FAIRE peaks are useful in identifying regulators of a biological process in an unbiased manner.

Siersbæk et al. (2011) recently used DNase-seq to investigate genome-wide change in open chromatin structure at various time points during 3T3-L1 differentiation. They reported a dramatic increase in the number of open chromatin sites in the first hours of differentiation. Such regions included what they termed "hot spots" that were bound by multiple adipogenic regulators, facilitating binding of PPAR $\gamma$ and $\mathrm{C} / \mathrm{EBP} \alpha$ during the late stage of differentiation. We found that the DNase I hypersensitive sites in 3T3-L1 cells on day 0 or 6 in the Siersbæk study significantly overlapped the FAIRE peaks on day 0 or 8 in our study (78.8\% and $80.9 \%$, respectively), suggesting that both methods detect similar open chromatin regions.

\section{REFERENCES}

Berg AH, Combs TP, Du X, Brownlee M, Scherer PE. 2001. The adipocytesecreted protein Acrp30 enhances hepatic insulin action. Nat Med 7: 947-953.

Bryne JC, Valen E, Tang MH, Marstrand T, Winther O, da Piedade I, Krogh A, Lenhard B, Sandelin A. 2008. JASPAR, the open access database of transcription factor-binding profiles: New content and tools in the 2008 update. Nucleic Acids Res 36: D102-D106.

Cantó C, Gerhart-Hines Z, Feige JN, Lagouge M, Noriega L, Milne JC, Elliott PJ, Puigserver P, Auwerx J. 2009. AMPK regulates energy expenditure by modulating $\mathrm{NAD}^{+}$metabolism and SIRT1 activity. Nature 458: 1056-1060.

Cantó C, Jiang LQ, Deshmukh AS, Mataki C, Coste A, Lagouge M, Zierath JR, Auxwerx J. 2010. Interdependence of AMPK and SIRT1 for metabolic adaptation to fasting and exercise in skeletal muscle. Cell Metab 11: 213-219.
Giresi PG, Kim J, McDaniell RM, Iyer VR, Lieb JD. 2007. FAIRE (formaldehyde-assisted isolation of regulatory elements) isolates active regulatory elements from human chromatin. Genome Res 17: 877-885.

Gupta RK, Arany Z, Seale P, Mepani RJ, Ye L, Conroe HM, Roby YA, Kulaga H, Reed RR, Spiegelman BM. 2010. Transcriptional control of preadipocyte determination by Zfp423. Nature 464: 619-623.

Handschin C, Spiegelman BM. 2008. The role of exercise and PGC1 $\alpha$ in inflammation and chronic disease. Nature 454: $463-469$.

Heintzman ND, Hon GC, Hawkins RD, Kheradpour P, Stark A, Harp LF, Ye Z, Lee LK, Stuart RK, Ching CW, et al. 2009. Histone modifications at human enhancers reflect global cell type-specific gene expression. Nature 459: 108-112.

$\mathrm{Hu}$ E, Liang P, Spiegelman BM. 1996. AdipoQ is a novel adipose-specific gene dysregulated in obesity. J Biol Chem 271: 10697-10703.

Iwabu M, Yamauchi T, Okada-Iwabu M, Sato K, Nakagawa T, Funata M, Yamaguchi M, Namiki S, Nakayama R, Tabata M, et al. 2010. Adiponectin and AdipoR1 regulate PGC1 $\alpha$ and mitochondria by $\mathrm{Ca}^{2+}$ and AMPK/SIRT1. Nature 464: $1313-$ 1319.

Kadowaki T, Yamauchi T, Kubota N, Hara K, Ueki K, Tobe K. 2006. Adiponectin and adiponectin receptors in insulin resistance, diabetes and metabolic syndrome-Adiponectin hypothesis. J Clin Invest 116: 1784-1792.

Kliewer SA, Umesono K, Noonan DJ, Heyman RA, Evans RM. 1992. Convergence of 9-cis retinoic acid and peroxisome proliferator signalling pathways through heterodimer formation of their receptors. Nature 358: 771-774.

Kubota N, Terauchi Y, Yamauchi T, Kubota T, Moroi M, Matsui J, Eto K, Yamashita T, Kamon J, Satoh H, et al. 2002. Disruption of adiponectin causes insulin resistance and neointimal formation. J Biol Chem 277: 25863-25866.

Maeda K, Okubo K, Shimomura I, Funahashi T, Matsuzawa Y, Matsubara K. 1996. cDNA cloning and expression of a novel adipose specific collagenlike factor, apM1 (adiPose most abundant gene transcript 1). Biochem Biophys Res Commun 221: $286-289$.

Mootha VK, Lindgren CM, Eriksson KF, Subramanian A, Sihag S, Lehar J, Puigserver P, Carlsson E, Ridderstråle M, Laurila $\mathrm{E}$, et al. 2003. PGC-1 $\alpha$-responsive genes involved in oxidative phosphorylation are coordinately downregulated in human diabetes. Nat Genet 34: 267-273.

Mootha VK, Handschin C, Arlow D, Xie X, St Pierre J, Sihag S, Yang W, Altshuler D, Puigserver P, Patterson N, et al. 2004. Err $\alpha$ and Gabpa/b specify PGC-1 $\alpha$-dependent oxidative phosphorylation gene expression that is altered in diabetic muscle. Proc Natl Acad Sci 101: 6570-6575.

Nakano Y, Tobe T, Choi-Miura NH, Mazda T, Tomita M. 1996. Isolation and characterization of GBP28, a novel gelatin-binding protein purified from human plasma. J Biochem 120: $803-$ 812.

Nielsen R, Pedersen TA, Hagenbeek D, Moulos P, Siersbæk R, Megens E, Denissov S, Børgesen M, Francoijs KJ, Mandrup S, et al. 2008. Genome-wide profiling of PPAR $\gamma: R X R$ and RNA polymerase II occupancy reveals temporal activation of distinct metabolic pathways and changes in RXR dimer composition during adipogenesis. Genes Dev 22: $2953-$ 2967.

Patti ME, Butte AJ, Crunkhorn S, Cusi K, Berria R, Kashyap S, Miyazaki Y, Kohane I, Costello M, Saccone R, et al. 2003. Coordinated reduction of genes of oxidative metabolism in humans with insulin resistance and diabetes: Potential role of PGC1 and NRF1. Proc Natl Acad Sci 100: 8466-8471.

Petersen KF, Dufour S, Befroy D, Garcia R, Shulman GI. 2004. Impaired mitochondrial activity in the insulin-resistant offspring of patients with type 2 diabetes. New Engl J Med 350: 664-671.

Scherer PE, Williams S, Fogliano M, Baldini G, Lodish HF. 1995. A novel serum protein similar to C1q, produced exclusively in adipocytes. J Biol Chem 270: 26746-26749. 
Schmidt SF, Jørgensen M, Chen Y, Nielsen R, Sandelin A, Mandrup S. 2011. Cross species comparison of $\mathrm{C} / \mathrm{EBP} \alpha$ and PPAR $\gamma$ profiles in mouse and human adipocytes reveals interdependent retention of binding sites. BMC Genomics 12: 152 .

Siersbæk R, Nielsen R, John S, Sung MH, Baek S, Loft A, Hager GL, Mandrup S. 2011. Extensive chromatin remodelling and establishment of transcription factor "hotspots" during early adipogenesis. EMBO J 30: 1459-1472.

Tontonoz P, Spiegelman BM. 2008. Fat and beyond: The diverse biology of PPAR $\gamma$. Annu Rev Biochem 77: 289-312.

Wakabayashi K, Okamura M, Tsutsumi S, Nishikawa NS, Tanaka T, Sakakibara I, Kitakami J, Ihara S, Hashimoto Y, Hamakubo T, et al. 2009. The peroxisome proliferator-activated receptor $\gamma /$ retinoid X receptor $\alpha$ heterodimer targets the histone modification enzyme PR-Set7/Setd8 gene and regulates adipogenesis through a positive feedback loop. Mol Cell Biol 29: 3544-3555.

Waki H, Nakamura M, Yamauchi T, Wakabayashi K, Yu J, Hirose-Yotsuya L, Take K, Sun W, Iwabu M, Okada-Iwabu M, et al. 2011. Global mapping of cell type-specific open chromatin by FAIRE-seq reveals the regulatory role of the NFI family in adipocyte differentiation. PLoS Genet 7: e1002311.

Wingender E, Chen X, Hehl R, Karas H, Liebich I, Matys V, Meinhardt T, Prüss M, Reuter I, Schacherer F. 2000. TRANSFAC: An integrated system for gene expression regulation. Nucleic Acids Res 28: $316-319$.
Wu Z, Puigserver P, Andersson U, Zhang C, Adelmant G, Mootha V, Troy A, Cinti S, Lowell B, Scarpulla RC, et al. 1999. Mechanisms controlling mitochondrial biogenesis and respiration through the thermogenic coactivator PGC-1. Cell 98: $115-124$

Yamauchi T, Kamon J, Waki H, Terauchi Y, Kubota N, Hara K, Mori Y, Ide T, Murakami K, Tsuboyama-Kasaoka N, et al. 2001. The fat-derived hormone adiponectin reverses insulin resistance associated with both lipoatrophy and obesity. Nat Med 7: 941-946.

Yamauchi T, Kamon J, Minokoshi Y, Ito Y, Waki H, Uchida S, Yamashita S, Noda M, Kita S, Ueki K, et al. 2002. Adiponectin stimulates glucose utilization and fatty-acid oxidation by activating AMP-activated protein kinase. Nat Med 8: 12881295.

Yamauchi T, Kamon J, Waki H, Imai Y, Shimozawa N, Hioki K, Uchida S, Ito Y, Matsui J, Eto K, et al. 2003a. Globular adiponectin protected $\mathrm{ob} / \mathrm{ob}$ mice from diabetes and apoE deficient mice from atherosclerosis. J Biol Chem 278: 2461-2468.

Yamauchi T, Kamon J, Ito Y, Tsuchida A, Yokomizo T, Kita S, Sugiyama T, Miyagishi M, Hara K, Tsunoda M, et al. 2003b. Cloning of adiponectin receptors that mediate antidiabetic metabolic effects. Nature 423: 762-769.

Yamauchi T, Nio Y, Maki T, Kobayashi M, Takazawa T, Iwabu M, Okada-Iwabu M, Kawamoto S, Kubota N, Kubota T, 2007. Targeted disruption of AdipoR1 and AdipoR2 causes abrogation of adiponectin binding and metabolic actions. Nat Med 13: $332-339$. 


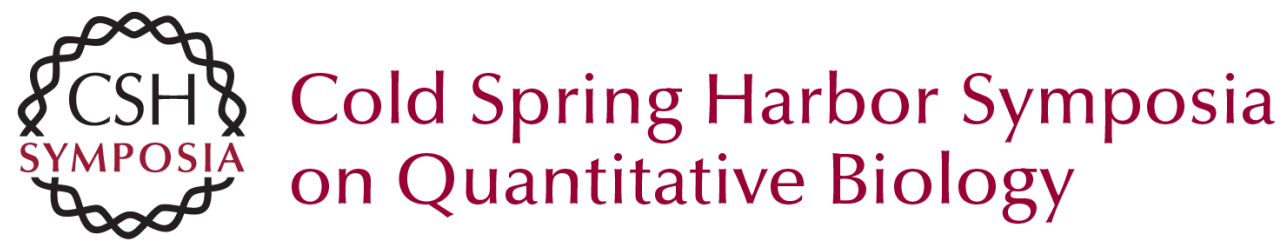

\section{Adiponectin, Adiponectin Receptors, and Epigenetic Regulation of Adipogenesis}

T. Kadowaki, T. Yamauchi, H. Waki, et al.

Cold Spring Harb Symp Quant Biol 2011 76: 257-265 originally published online April 4, 2012 Access the most recent version at doi:10.1101/sqb.2012.76.010587

References This article cites 33 articles, 9 of which can be accessed free at: http://symposium.cshlp.org/content/76/257.full.html\#ref-list-1

License

Email Alerting Receive free email alerts when new articles cite this article - sign up in Service the box at the top right corner of the article or click here. 